\title{
Retarded dipole-dipole dispersion interaction potential for helium
}

\section{Citation}

Jamieson, M. J., G. W. F. Drake, and A. Dalgarno. 1995. "Retarded Dipole-Dipole Dispersion Interaction Potential for Helium." Physical Review A 51 (4): 3358-61. https://doi.org/10.1103/ physreva.51.3358.

\section{Permanent link}

http://nrs.harvard.edu/urn-3:HUL.InstRepos:41417405

\section{Terms of Use}

This article was downloaded from Harvard University's DASH repository, and is made available under the terms and conditions applicable to Other Posted Material, as set forth at http:// nrs.harvard.edu/urn-3:HUL.InstRepos:dash.current.terms-of-use\#LAA

\section{Share Your Story}

The Harvard community has made this article openly available.

Please share how this access benefits you. Submit a story.

Accessibility 


\title{
Retarded dipole-dipole dispersion interaction potential for helium
}

\author{
M. J. Jamieson \\ Department of Computing Science, University of Glasgow, Glasgow G12 8QQ, United Kingdom \\ G. W. F. Drake \\ Department of Physics, University of Windsor, Windsor, Ontario, Canada N9B 3P4
}

A. Dalgarno

Harvard-Smithsonian Center for Astrophysics, Cambridge, Massachusetts 02138

(Received 16 September 1994)

\begin{abstract}
The retarded dipole-dipole dispersion interaction potential in helium is evaluated from a set of very accurate effective dipole transition frequencies and oscillator strengths already obtained from a variational calculation. The asymptotic form changes from the inverse sixth to the inverse seventh power of the nuclear separation as the atoms move apart. Simple representations of the potential are given for use in scattering and structure calculations.
\end{abstract}

PACS number(s): $34.50 .-\mathrm{s}, 34.20 . \mathrm{Cf}$

\section{INTRODUCTION}

Two widely separated atoms influence each other mainly through the long-range dipole-dipole dispersion interaction. In the absence of relativistic corrections, the interaction potential varies asymptotically as $R^{-6}$ where $R$ is the internuclear distance. When the atoms are sufficiently far apart that the time for an electromagnetic signal to travel from one to the other is comparable to the period of the lowest allowed dipole transition of either, retardation must be taken into account and the asymptotic variation of the potential becomes $R^{-7}$. The relativistic expression for the potential was first given by Casimir and Polder [1]. Retardation is a very small long-range effect. It is negligible in scattering calculations and unobservable in experiments at temperatures greater than a few degrees $\mathbf{K}$. However, the rapidly expanding experimental field of ultra-low-temperature scattering measurements [2-9] may lead to detectable effects and has introduced the need for very low energy scattering and structure calculations which include retardation; a recent low-temperature experiment involving helium may be the first direct observation of retardation effects in neutral atom interactions [10]. The retarded potential for a pair of ground-state helium atoms has been calculated by Getzin and Karplus [11], Langhoff [12], and Luo et al. [13], using approximate dynamic polarizability data [14]. Below we present details of a very accurate calculation of the retarded potential for a pair of ground-state helium atoms, based on recent precise values of the polarizability [15].

\section{CALCULATions}

In atomic units (a.u.), which will be used throughout, the potential $V(R)$ is $[1,13]$

$$
\begin{aligned}
V(R)=-\frac{1}{R^{6}} \frac{1}{\pi} \int_{0}^{\infty} & d u \beta^{2}(i u) e^{-2 u R \alpha} \\
\times[ & (u R \alpha)^{4}+2(u R \alpha)^{3}+5(u R \alpha)^{2} \\
& +6 u R \alpha+3],
\end{aligned}
$$

where $\beta(i u)$ is the dipole polarizability at the imaginary frequency $i u$ and $\alpha=1 / 137.0359895$ is the fine structure constant. The value of $\beta(i u)$ was obtained by analytically continuing the expression for the dynamic polarizability $\beta(\omega)$, at frequency $\omega$, in terms of a set of $N$ effective dipole transition frequencies $\omega_{i}$ and oscillator strengths $f_{i}$ which were obtained from a very precise variational calculation of Drake [15]

$$
\beta(\omega)=\sum_{i=1}^{N} \frac{f_{i}}{\omega_{i}^{2}-\omega^{2}},
$$

where $N=197$ in these calculations. The method yields a lower bound to the magnitude of $V(R)$.

At small values of $R$

$$
V(R) \approx \frac{-C_{6}}{R^{6}},
$$

where

$$
C_{6}=\frac{3}{\pi} \int_{0}^{\infty} d u \beta^{2}(i u) .
$$

At large values of $R$

$$
V(R) \approx \frac{-C_{7}}{R^{7}},
$$

where

$$
C_{7}=\frac{23 \beta^{2}(0)}{4 \pi \alpha} .
$$

With the variable of integration changed to $x=2 u R \alpha$, the potential is 
TABLE I. Retarded dipole-dipole dispersion interaction potential in helium.

\begin{tabular}{|c|c|c|c|c|c|}
\hline$R$ (bohr) & $-V(R) R^{6} / C_{6}$ & $-V(R) R^{7} / C_{7}$ & $R$ (bohr) & $-V(R) R^{6} / C_{6}$ & $-V(R) R^{7} / C_{7}$ \\
\hline 0.0 & 1.0000000 & 0.0000000 & 0.2 & 0.9999990 & 0.0006089 \\
\hline 0.4 & 0.9999962 & 0.0012178 & 0.6 & 0.9999914 & 0.0018267 \\
\hline 0.8 & 0.9999847 & 0.0024356 & 1.0 & 0.9999762 & 0.0030445 \\
\hline 1.2 & 0.9999658 & 0.0036534 & 1.4 & 0.9999537 & 0.0042622 \\
\hline 1.6 & 0.9999397 & 0.0048710 & 1.8 & 0.9999239 & 0.0054798 \\
\hline 2.0 & 0.9999063 & 0.0060886 & 2.2 & 0.9998869 & 0.0066973 \\
\hline 2.4 & 0.9998659 & 0.0073060 & 2.6 & 0.9998430 & 0.0079146 \\
\hline 2.8 & 0.9998185 & 0.0085232 & 3.0 & 0.9997923 & 0.0091318 \\
\hline 3.5 & 0.9997193 & 0.0106530 & 4.0 & 0.9996360 & 0.0121738 \\
\hline 4.5 & 0.9995426 & 0.0136943 & 5.0 & 0.9994393 & 0.0152143 \\
\hline 6.0 & 0.9992037 & 0.0182529 & 7.0 & 0.9989308 & 0.0212892 \\
\hline 8.0 & 0.9986219 & 0.0243230 & 9.0 & 0.9982784 & 0.0273540 \\
\hline 10.0 & 0.9979016 & 0.0303818 & 20.0 & 0.9925364 & 0.0604369 \\
\hline 30.0 & 0.9848591 & 0.0899542 & 40.0 & 0.9754952 & 0.1187985 \\
\hline 50.0 & 0.9648867 & 0.1468833 & 60.0 & 0.9533590 & 0.1741541 \\
\hline 70.0 & 0.9411588 & 0.2005797 & 80.0 & 0.9284765 & 0.2261450 \\
\hline 90.0 & 0.9154615 & 0.2508468 & 100.0 & 0.9022318 & 0.2746908 \\
\hline 110.0 & 0.8888816 & 0.2976889 & 120.0 & 0.8754865 & 0.3198576 \\
\hline 130.0 & 0.8621069 & 0.3412168 & 140.0 & 0.8487917 & 0.3617888 \\
\hline 150.0 & 0.8355801 & 0.3815973 & 160.0 & 0.8225034 & 0.4006671 \\
\hline 170.0 & 0.8095868 & 0.4190235 & 180.0 & 0.7968500 & 0.4366919 \\
\hline 190.0 & 0.7843086 & 0.4536977 & 200.0 & 0.7719745 & 0.4700661 \\
\hline 300.0 & 0.6610692 & 0.6038015 & 400.0 & 0.5719587 & 0.6965474 \\
\hline 500.0 & 0.5007741 & 0.7623210 & 600.0 & 0.4434520 & 0.8100725 \\
\hline 700.0 & 0.3967342 & 0.8455195 & 800.0 & 0.3581683 & 0.8723748 \\
\hline 900.0 & 0.3259355 & 0.8931001 & 1000.0 & 0.2986835 & 0.9093630 \\
\hline 2000.0 & 0.1597728 & 0.9728791 & 3000.0 & 0.1081080 & 0.9874273 \\
\hline 4000.0 & 0.0815234 & 0.9928152 & 5000.0 & 0.0653864 & 0.9953670 \\
\hline 6000.0 & 0.0545654 & 0.9967693 & 7000.0 & 0.0468103 & 0.9976204 \\
\hline 8000.0 & 0.0409818 & 0.9981751 & 9000.0 & 0.0364422 & 0.9985565 \\
\hline 10000.0 & 0.0328069 & 0.9988298 & 20000.0 & 0.0164179 & 0.9997067 \\
\hline 30000.0 & 0.0109470 & 0.9998696 & 40000.0 & 0.0082107 & 0.9999266 \\
\hline 50000.0 & 0.0065688 & 0.9999530 & 60000.0 & 0.0054740 & 0.9999674 \\
\hline 70000.0 & 0.0046921 & 0.9999760 & 80000.0 & 0.0041056 & 0.9999817 \\
\hline 90000.0 & 0.0036494 & 0.9999855 & 100000.0 & 0.0032845 & 0.9999883 \\
\hline$\infty$ & 0.0000000 & 1.0000000 & & & \\
\hline
\end{tabular}

$$
\begin{aligned}
V(R)=\frac{-1}{R^{7}} \frac{1}{2 \pi \alpha} \int_{0}^{\infty} & d x \beta^{2}\left[\frac{i x}{2 R \alpha}\right] e^{-x} \\
& \times\left(\frac{x^{4}}{16}+\frac{x^{3}}{4}+\frac{5 x^{2}}{4}+3 x+3\right)
\end{aligned}
$$

and the polarizability is

$$
\beta(i u)=\sum_{i=1}^{N} \frac{f_{i}}{\omega_{i}^{2}+u^{2}} .
$$

Thus retardation becomes important if the time for propagating an electromagnetic signal between the atoms $(R \alpha)$ is comparable with the period of the lowest dipole transition $\left(1 / \omega_{1}\right)$.

The quadrature was evaluated numerically by application of Romberg's method to trapezium rule estimates obtained with different step lengths. The range of $x$ was taken as $0-35 ; e^{-35} \approx 6 \times 10^{-16}$ and the polynomial part of the integrand is approximately $10^{5}$ at $x=35$. The range was divided into 35 subranges of unit length and the contribution of each was evaluated to within a rela- tive accuracy of $10^{-9}$. This partitioning reduced the effect of the modulation by the negative exponential factor; with a single partition the quadrature is forced into taking an unnecessarily large number of steps.

The value of $C_{6}$ was calculated directly from the nonretarded expression. We obtained a value of 1.460978 a.u. To provide a check on the numerical procedures, $C_{6}$ was also evaluated by extrapolating to $R=0$ the values of $R^{6} V(R)$ calculated from the retardation expression. The value of $C_{7}$ was obtained from the static dipole polarizability. For the static dipole polarizability,

TABLE II. Coefficients for analytic fits to potential.

\begin{tabular}{lrr}
\hline \hline$i$ & \multicolumn{1}{c}{$a_{i}$} & \multicolumn{1}{c}{$b_{i}$} \\
\hline 0 & $-1.62343 \times 10^{-3}$ & $8.82506 \times 10^{-2}$ \\
1 & $2.22097 \times 10^{-3}$ & $3.81846 \times 10^{-2}$ \\
2 & $-1.17323 \times 10^{-3}$ & $-1.72421 \times 10^{-3}$ \\
3 & $3.00012 \times 10^{-4}$ & \\
4 & $-1.05512 \times 10^{-5}$ & $4.74897 \times 10^{-7}$ \\
\hline \hline
\end{tabular}




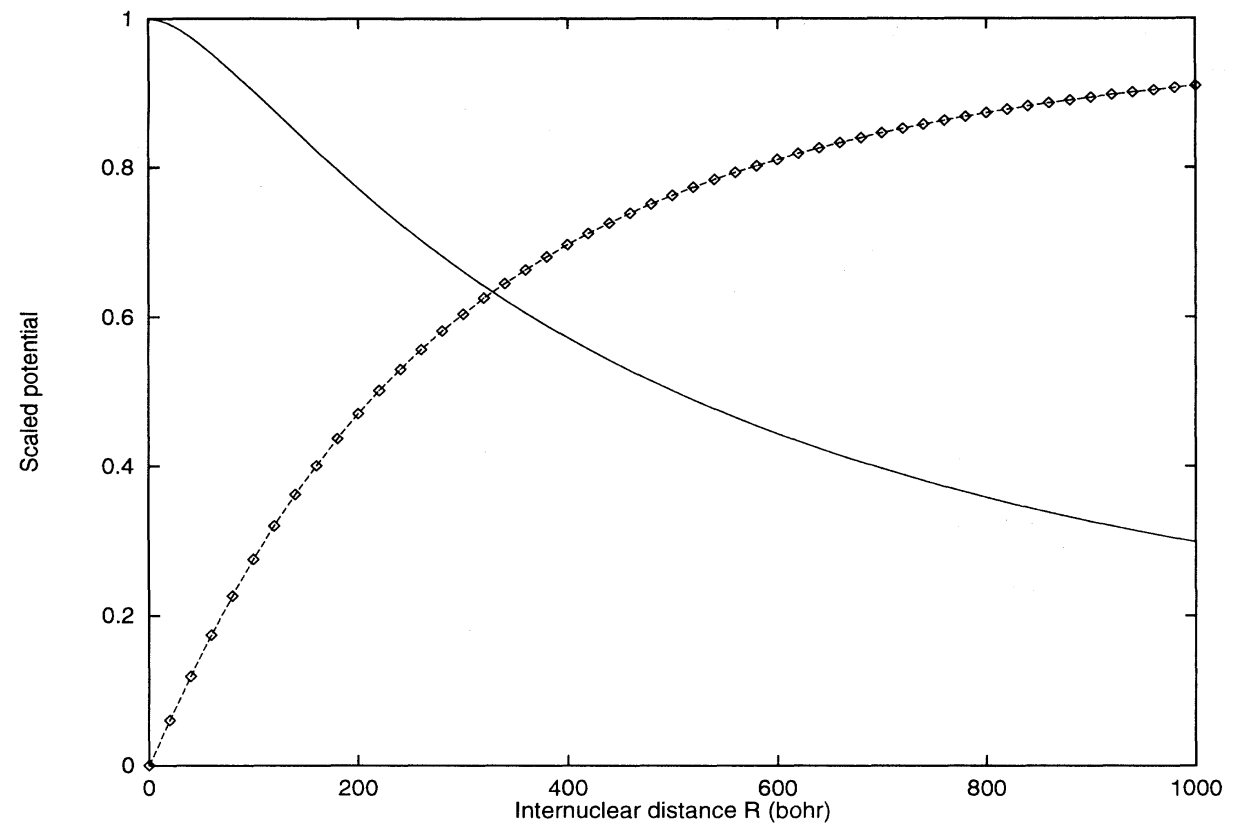

$\begin{array}{cccc}\text { FIG. } 1 . & \text { Full curve: } \\ -R^{6} V(R) / C_{6}, & \text { dashed } & \text { curve: } \\ -R^{7} V(R) / C_{7} . & & \end{array}$

we obtained a value of 1.383192 a.u. and for $C_{7}$ we obtained 479.8634 a.u. The results for $C_{6}$ and $C_{7}$ were calculated in the limit of infinite nuclear mass ignoring relativistic corrections and are accurate to the number of figures quoted. Finite mass corrections would modify the static polarizability to

$$
\beta(0)=1.383192+0.35626 \mu / M,
$$

expressed in units of the cube of the reduced mass Bohr radius $a_{M}^{3}$, where $\mu / M$ is the ratio of the reduced electron mass to the nuclear mass. This value of $\beta(0)$ is in agreement with the calculations of Bhatia and Drachman [16]. Further relativistic corrections may be of the same order and are also negligible. A separate calculations with the four term data of Chan and Dalgarno [14] showed that the potential is insensitive to the details of the effective dipole transitions, as noted by Luo et al. [13] in their calculations of the retarded potential. The uncertainty in the results in Table I should be less than one in the fourth significant figures following the leading zeros or nines.

We derived analytic fits to the potential in the range 10-200 bohr. The dispersion potential is not appropriate for $R<10$. For values at $R>200$, interpolation of the tabulated values can be used. The analytic fits for $R$ in the range $10-100$ bohr are

$$
V(R)=-\frac{C_{6}}{R^{6}}[1-f(R)],
$$

where

$$
f(R)=a_{0}+a_{1} R^{1 / 2}+a_{2} R+a_{3} R^{3 / 2}+a_{4} R^{2}
$$

and for $R$ in the range 100-200

$$
V(R)=-\frac{C_{6}}{R^{6}} \frac{1+g(R)}{1.2+0.8 R C_{6} / C_{7}},
$$

where

$$
g(R)=b_{0}+b_{1} R^{1 / 2}+b_{2} R+b_{4} R^{2},
$$

the values of $a_{i}$ and $b_{i}$ being given in Table II. The functions $f(R)$ and $g(R)$ were determined by least-squares fits to the calculated quantities $-\left[1+V(R) R^{6} / C_{6}\right]$ and $-\left[1+1.2\left(V(R) R^{6} / C_{6}\right)+0.8\left(V(R) R^{7} / C_{7}\right)\right]$, respectively; the latter quantity was found, by numerical experiment, to vary slowly over the range 100-200. The forms of the expansions of $f(R)$ and $g(R)$ were also determined by numerical experiment. Note the absence of a term $R^{3 / 2}$ in the expansion of $g(R)$; a better fit was obtained by omitting it. The analytic expressions reproduce the potential to within a relative accuracy of $10^{-5}$.

We present our results graphically in Fig. 1 which shows how the effect of retardation changes the $R$ dependence from $R^{-6}$ to $R^{-7}$ as $R$ increases. Retardation becomes apparent as the signal propagation time approaches the period of the lowest dipole transition.

O'Carroll and Sucher [17] advanced the formula

$$
-V(R) R^{6} / C_{6}=(2 / \pi) \tan ^{-1}(d / R),
$$

where $d$ is a characteristic length

$$
d=(\pi / 2) C_{7} / C_{6}
$$

as a representation of the retarded interaction that is free of disposable parameters. Langhoff [12] found that it yielded results within a few percent of his calculated values. We find that it underestimates our values by less than $3 \%$ for $R<260$ and overestimates them by less than $2 \%$ for larger $R$, becoming exact in the asymptotic limit of large $R$.

\section{ACKNOWLEDGMENTS}

This work was supported by the Division of Chemical Sciences, Office of Basic Energy Sciences, Office of Energy Research, U. S. Department of Energy. 
[1] H. B. G. Casimir and D. Polder, Phys. Rev. 73, 360 (1948); G. Feinberg, J. Sucher, and C. K. Au, Phys. Rep. 180, 84 (1989); E. A. Power and T. Thirunamachandran, Phys. Rev. A 48, 4761 (1993); Chem. Phys. 171, 1 (1993).

[2] M. Prentiss, A. Cable, J. Bjorkholm, S. Chu, E. Rachard, and D. Pritchard, Opt. Lett. 13, 452 (1988).

[3] F. L. Gould, P. D. Lett, P. S. Julienne, W. D. Phillips, H. R. Thorsheim, and J. Weiner, Phys. Rev. Lett. 60, 788 (1988).

[4] D. Sesko, T. Walker, C. Monroe, A. Gallagher, and C. Wiemar, Phys. Rev. Lett. 63, 961 (1989).

[5] P. D. Lett, P. S. Jessen, W. D. Phillips, S. L. Rolston, C. I. Westbrook, and P. L. Gould, Phys. Rev. Lett. 67, 2139 (1991).

[6] P. D. Lett, K. Helmerson, W. D. Phillips, L. P. Ratliff, S. L. Rolston, and M. E. Wagshul, Phys. Rev. Lett. 71, 2200 (1993).

[7] J. D. Miller, R. A. Cline, and D. J. Heinzen, Phys. Rev. Lett. 71, 2204 (1993).
[8] F. Luo, G. Kim, G. F. Giese, and W. R. Gentry, J. Chem. Phys. 99, 10084 (1993).

[9] J. C. Mester, E. S. Meyer, M. W. Reynolds, T. E. Huber, Z. Zhao, B. Freedman, J. Kim, and I. F. Silvera, Phys. Rev. Lett. 71, 1343 (1993).

[10] F. Luo, G. C. McBane, G. Kim, C. F. Giese, and W. R. Gentry, J. Chem. Phys. 98, 3564 (1993).

[11] P. M. Getzin and M. Karplus, J. Chem. Phys. 53, 2100 (1971).

[12] P. W. Langhoff, Chem. Phys. Lett. 12, 223 (1971).

[13] F. Luo, G. Kim, G. C. McBane, C. F. Giese, and W. R. Gentry, J. Chem. Phys. 98, 9687 (1993).

[14] Y. M. Chan and A. Dalgarno, Proc. Phys. Soc. London 86, 777 (1965).

[15] G. W. F. Drake (unpublished).

[16] A. K. Bhatia and R. J. Drachman, J. Phys. B 27, 1299 (1994).

[17] M. O’Carroll and J. Sucher, Phys. Rev. 187, 85 (1969). 